\title{
Factores que influyen en el desempeño matemático de estudiantes universitarios iniciales
}

\section{Raúl González}

Universidad de Lima

Lima, Perú

Fueron examinados 67 estudiantes universitarios iniciales en tres áreas de sus competencias matemáticas: esquemas operatorios lógicos, habilidades algoritmicas y de cálculo y capacidad de lectura de problemas matemáticos. Se analizó el papel de factores grupales y se trazó el perfil diferencial de competencias entre los estudiantes de alto y bajo rendimientos. Identificamos además tres carencias comunes a ambos grupos: en operaciones lógico-formales, debilidad probabilística, combinatoria y distributiva; en cálculo, debilidad metacognitiva por escasas estimaciones anticipatorias; y en lectura de problemas, dificultad en hallar los enunciados relevantes.

esquemas operatorios / habilidades algorítmicas / razonamiento lógico-concreto / pensamiento matemático

\section{Influency factors in mathematical performance of first-year college students}

First year college students were assessed in three areas of mathematical competence: logical operational schemes, algorithmic and circle abilities and reading ability of mathematical problems. Group factors were analyzed and a profile developed between achievers and underachievers. Three needs common to both groups were identified: in formal logical operations, probabilistic, combining and distributive weaknesses; in calculus, weak metacognition due to poor anticipatory estimations, and in the reading of problems, difficulty finding the relevant statements.

operational schemes / algorithmic abilities / concrete logical reasoning / mathematical thinking 
Es un criterio ampliamente generalizado que la enseñanza-aprendizaje de las matemáticas escolares no alcanza adecuados niveles de realización (Skemp, 1999; González, 1998). Probablemente el núcleo del problema esté en el proceso de formación de profesores para los niveles primario, secundario y superior. Resolver el problema de qué y cómo enseñar a los futuros profesores tiene como condición el saber qué y cómo enseñar matemáticas a los escolares. Si no existe una idea clara de los factores que condicionan los rendimientos escolares en una área determinada será muy difícil, por no decir imposible, elaborar sugerencias y recomendaciones realistas para mejorar el proceso de enseñanza-aprendizaje. El docente primario, como enseñante del ciclo básico en el que las matemáticas ocupa un papel relevante, el docente secundario que ya es un profesor especializado en el área y el docente universitario, principalmente el de las asignaturas de Estudios Generales y el que apoya en cursos de matemáticas a facultades y escuelas de especialidades no matemáticas como las humanidades, requieren una formación pedagógica que los ponga en condiciones de realizar un trabajo educativo cuya productividad se manifieste en el rendimiento académico de sus estudiantes. El propósito de nuestro trabajo es colaborar con un insumo de información pertinente a la enseñanza de las matemáticas de nivel universitario, tratando de identificar algunas condicio- nes o factores que determinan el rendimiento desde el punto de vista de las habilidades o estrategias que ponen en marcha diferencialmente los buenos y los malos alumnos de los cursos universitarios del nivel de Estudios Generales. Nuestro propósito es aproximarnos a trazar un perfil de cuáles son las habilidades o estrategias matemáticas que determinan las diferencias en los rendimientos académicos de los alumnos recién ingresantes a la universidad. En la literatura científica sobre psicología del aprendizaje matemático pueden distinguirse tres tipos de estrategias, que vamos a denominar esquemas operatorios, procedimientos de cálculo $\mathrm{y}$ estrategias resolutorias de problemas.

\section{LOS ESQUEMAS OPERATORIOS}

Piaget $(1967,1983)$ definió las operaciones mentales como acciones simbólicas interiorizadas y reversibles. Son acciones simbólicas en el doble sentido de que son transformaciones que se ejecutan sobre los símbolos de los objetos y no sobre los objetos mismos y que esas transformaciones se soportan en sus propios significantes simbólicos. La operación de suma, por ejemplo, opera transformaciones sobre los números simbolizados en los numerales y se soporta en sus propios significantes como el logograma +. Las operaciones son acciones interiorizadas; es decir, se desarrollan en el plano de la actividad mental del sujeto y no en el plano de la conducta. Finalmente, las 
operaciones son reversibles, es decir, a toda operación en una dirección le corresponde una operación en la dirección contraria que anula la operación realizada. Así a la operación de suma se opone la operación de resta haciendo reversible la primera operación, a $5+3$ $=8$ le corresponde en reversión 8-3 = 5 , en el cual se regresa al símbolo numérico 5 que fue objeto de la transformación aditiva inicial.

Las operaciones -siguiendo a Piagetse desarrollan en el proceso evolutivo en tres etapas netamente diferenciadas: preoperatoria, de 2 a 7 años; lógicoconcreta: de 7 a 12 años; y lógico-formal, de 12 a 16 años. Las características generales de las etapas piagetanas están definidas en la etapa preoperatoria, por una diferenciación y articulación muy fluida de la estructura simbólica significante-significado; pero, las transformaciones que se realizan con los símbolos carecen de reversibilidad y de restricciones lógicas. La etapa lógico-concreta está determinada por la emergencia plena de las operaciones en el plano de las representaciones que le permiten estructurar el presente en función del pasado sin las deformaciones, dislocaciones ni contradicciones del niño preoperatorio. La etapa lógico-formal se caracteriza por operaciones sobre lo posible, en la que lo real emerge como uno de sus casos. Frente a un problema se preven todas las relaciones de posible pertinencia y se determina por actuación empírica y análisis crítico la validez real de algunas de las posibles relaciones. Es un pensamiento hipotético-deductivo, proposicional y probabilístico. El desarrollo operatorio genera mecanismos mentales que hacen posible tres modos de procesamiento típicos: a) aprehensión simultánea simbólica e interna, en una síntesis única de una serie completa de hechos separados; b) reflexión sobre la organización de los propios actos con orientación contemplativa, además de activa; y c) independización de los actos presentes de los objetos concretos del mundo real, transitando a manipular simbólicamente entidades no tangibles.

En el plano de las matemáticas las operaciones se desarrollan a partir de los siete años, cuando el niño supera el estado denominado preconservante, caracterizado así cuando los objetos en el marco de transformaciones percibidas no conservan para el niño la cantidad de sustancia. Es, posteriormente, a los seis años que el niño inicia el dominio de acciones como correspondencias biunívocas, relaciones de equivalencia, adición de clases, composición aditiva, correspondencia ordinal y conservación de la cantidad. Esta última puede ser continua o discontinua e inicialmente referida a la sustancia, posteriormente habrá conservación del peso (nueve años) y del volumen (11 años). Coincidente con el desarrollo en el plano de las transformaciones numéricas se inicia el desarrollo de las transformaciones geométricas: construcción de 
la medida, conservación de la longitud, determinación de lugares geométricos y geometrización del espacio. Entre los siete y 12 años el niño es capaz de actuar bajo restricciones lógicas y manipular bajo tales condiciones sus representaciones: clasificaciones jerárquicas, seriación ordenada y conservación de invariantes de cantidad, peso y volumen. Es en este escenario mental de los siete a 12 años que se configuran las operaciones aritméticas y métricas.

Las operaciones aritméticas consisten en el grupo aditivo de números enteros con propiedades de composición, asociatividad, inverso, identidad e iteración; el otro grupo es el multiplicativo de números enteros con análogas propiedades de grupo. El grupo es un sistema de transformaciones con composición, asociatividad, inversión o reversibilidad. La iteración convierte los elementos lógicos intensivos en cuantificables.

Las operaciones métricas de esta misma etapa lógico-concreta consisten en la medición de relaciones partes a todo, en la que las partes se conviertan en unidades iterables a las que se les aplican números. Depende de operaciones infralógicas de partición aplicadas a los objetos, en virtud de las cuales el objeto es una entidad única, cuyas partes no permanecen ni independientes ni separadas cuando componen un todo y de que la constitución por síntesis del objeto requiere de la proximidad de las partes. Las operaciones métricas son partitivas y sintetizadoras en correspondencia con la adición y sustracción de clases.

Es en la edad de 12 a 16 años, la etapa lógico-formal, en la que se constituyen los esquemas operatorios. Las operaciones lógico-concretas tienen como punto de partida lo real, están ancladas como islotes funcionales aquí y ahora. Las operaciones lógico-formales elaboran lo potencial y lo posible como distinto de lo real. Se desarrollan estrategias hipotético-deductivas y las entidades más importantes en el razonamiento no son las representaciones de eventos concretos sino el pensamiento proposicional que se genera por vínculos intraproposicionales e interproposicionales, entre proposiciones resultantes de las operaciones concretas. Son operaciones sobre operaciones, $\mathrm{u}$ operaciones de segundo grado.

En este nuevo escenario mental lógico-formal, emergen -en el plano de las matemáticas- cuatro esquemas operatorios formales, que son estrategias y procedimientos complejos de transformación y procesamiento mental. Los esquemas lógico-matemáticos más importantes son los esquemas de combinaciones, proporciones, correlación y de distribución. El esquema de combinaciones está caracterizado por el análisis cuantificado, ordenado y exhaustivo de todas las relaciones posibles entre procesos o eventos determinados. El esquema de proporciones determina relaciones ordenadas, directas o inver- 
sas, entre eventos definidos por una razón cuantitativa. El esquema de correlación determina la predictibilidad de un evento en función del acaecimiento de otro con el cual mantiene una relación de asociación cuya posibilidad es cuantificada. Finalmente, el esquema de distribución prevé la asimetría de eventos en forma de campana gaussiana, con una zona central estándar y zonas extremas más reducidas y simétricas (Inhelder y Piaget).

\section{Procedimientos de cálculo}

El cálculo es el conjunto de algoritmos y estrategias de cómputo, mediante los cuales se manipulan los números y sus símbolos. Desde una perspectiva psicopedagógica, interesa la precisión o error con la que el sujeto manipula los números. Cuando el sujeto afronta una tarea moviliza -además de los conocimientos pertinentes- una serie de conocimientos prerrequisitos. Los errores que sólo se dan en la tarea-meta se deben a estrategias incorrectas. Los errores en la tarea-meta y en prerrequisitos involucran las habilidades de base para el cálculo de los sujetos. Algunos indicadores importantes con respecto al cálculo que figura en la literatura (Gagné, Resmeck) son, entre otros, el dominio posicional de los números, sus transformaciones posicionales y la comprensión del ábaco; las estimaciones como aproximaciones tentativas a los resultados y que funcionan como representaciones de control, para evaluar los re- sultados como válidos o no, por discrepancias al comparar con la anticipación conjetural; otros dos aspectos importantes del número son tanto su relación con la recta como la comprensión de las fracciones, aspectos tras los que subyace una lógica común.

\section{RESOLUCIÓN DE PROBLEMAS}

La organización del conocimiento matemático resultante de la aplicación de los esquemas operatorios al propio conocimiento y a la experiencia matemática se refleja en la solución de problemas. En este aspecto los factores fundamentales están enfocados en la comprensión de los enunciados narrativos identificando las características matemáticas de dichos enunciados y los aspectos relevantes desde el punto de vista de su pertinencia para la solución. Otro segundo aspecto, muy importante, radica en la articulación y congruencia de lo que podríamos denominar la "traducción" de problemas a ecuaciones y de ecuaciones a problemas. Es decir, la puesta en relación significativa de dos lenguajes: el natural y el matemático (González, 1998).

\section{FACTORES GRUPALES}

En una investigación anterior sobre comprensión lectora (González, 1998), definimos la necesidad de explorar la pertinencia de algunas variables tales como edad, género, procedencia escolar y algunos otros más que configuran 
subgrupos poblacionales importantes en la universidad. La exploración de estas variables tiene como objetivo identificar tanto su papel predictor de rendimientos como las diferencias significativas que pueden existir entre tales subgrupos. En el caso de la lectura fue identificada como variable con alto valor predictivo el ingreso o no, inmediato, a la universidad.

\section{MÉTodo}

\section{Muestra}

Se exploró una población de 67 estudiantes de los ciclos iniciales de Estudios Generales de una universidad privada. Los sujetos de cada grupo correspondieron, respectivamente, 27 al de alto rendimiento y 40 al de bajo rendimiento. La caracterización de alto o bajo rendimiento fue definida por las oficinas pedagógicas pertinentes sobre la base del rendimiento académico de los alumnos. Debe anotarse que los alumnos del grupo de rendimiento alto, cuando fueron convocados a la aplicación del instrumento, actuaron en general con espíritu de colaboración y con comodidad. A los integrantes del grupo de bajo rendimiento fue difícil reunirlos pues rehuyeron la convocatoria inicial y, durante la aplicación del instrumento, muchos de ellos dejaron de colaborar mientras otros manifestaban su incomodidad y aversión frente a la realización de tareas matemáticas. Cuando hemos explorado otras áreas de rendimiento, como comprensión lectora, jamás se habían presentado situaciones de elevado rechazo como en esta ocasión, al explorarse los desempeños matemáticos. En la tabla $N^{0} 1$ se presenta a la población explorada clasificada, además de la dicotomización en alto y bajo por nivel de rendimiento, en nueve dimensiones que nos permitieron organizar subgrupos pertinentes. Los agrupamientos por rasgos determinaron una población con las siguientes características dominantes: edad entre 18 y 20 años (42 sujetos), masculinos (46 sujetos), estudiantes de ciencias (48 sujetos), procedentes de Lima (56 sujetos), sin repitencia escolar (59 sujetos), de padres con educación superior por lo menos uno de ellos (57 sujetos), provenientes de colegios particulares (62 sujetos) y en categorías de pago DE-F (49 sujetos). Sólo en el agrupamiento por ingreso a la universidad la distribución fue prácticamente igual: inmediato (33 sujetos) y posterior (34 sujetos). El carácter de nuestra muestra ha sido, obviamente, no probabilístico y basado en criterio de rendimiento.

\section{Instrumento}

En la tabla $\mathrm{N}^{\circ} 2$ presentamos la estructura de las 63 preguntas que hiciéramos en forma de cuestionario escrito (solicitar copia al editor): Las letras 0 , C y $\mathrm{P}$ representan la estructura básica del instrumento que pretende explorar operaciones lógico-matemáticas y es- 
quemas operatorios (0), procedimientos y habilidades en algoritmos y cálculo (C) y encaramiento a planteamientos de problemas $(\mathrm{P})$. Cada número romano rotula una dimensión diferenciada dentro de cada una de las áreas exploradas, dimensión a la que se le asignó tres preguntas, calificada cada una con un punto. Hemos denominado "ítem" al grupo de tres preguntas correspondientes a una dimensión.

Las 24 preguntas del rubro O, operaciones, se agrupan en ocho dimensiones:

- 0-I.- Organización del conocimiento. Explora las redes clasificatorias (operaciones de clases) a través de sencillas preguntas de agrupamiento.

- 0-II.- Operaciones aritméticas. Busca explorar el reconocimiento de las aplicaciones de operaciones aritméticas simples.

- 0-IV.- Lectura de gráficos. Se propone explorar las operaciones de medición que relaciona dimensiones cuantificables de estructuras geométricas con eventos reales.

- 0-XXI.- Seriación. Explora las operaciones de ordenamiento mayormenor o menor-mayor con diverso tipo de números. La base operativa de estas cuatro operaciones es lógico-concreta: clasificación, seriación, numeración y seriación en el modelo piagetano.

Las otras cuatro operaciones exploradas corresponden a los cuatro esquemas operatorios lógico-formales.
- 0-IV.- Combinaciones. Es la exhaustiva puesta en relaciones pertinentes de objetos y eventos determinados.

- 0 -XII.- Probabilidades. En términos piagetanos, la medida de lo posible.

- 0-XVI.- Proporciones. Son relaciones cuantitativas que conservan una constante de razón.

- 0-V.- Distribuciones, caracterizadas por la curva gaussiana.

Tabla $N^{\circ} 1$

Muestra distribuida por rasgos poblacionales

\begin{tabular}{|c|c|c|}
\hline \multirow[t]{3}{*}{ Nivel de rendimiento } & Alto & 27 \\
\hline & Bajo & 40 \\
\hline & Menos de & 18 \\
\hline \multirow[t]{2}{*}{ Edad } & $18-20$ & 42 \\
\hline & Más de 20 & 7 \\
\hline \multirow{2}{*}{ Sexo } & Masculino & 46 \\
\hline & Femenino & 21 \\
\hline \multirow{3}{*}{ Carrera profesional } & Ciencias & 48 \\
\hline & Letras & 19 \\
\hline & Lima & 56 \\
\hline \multirow[t]{2}{*}{ Lugar de nacimiento } & Provincia & 9 \\
\hline & Otro país & 2 \\
\hline \multirow{2}{*}{ Repitencia escolar } & Sí & 8 \\
\hline & No & 59 \\
\hline \multirow{2}{*}{ Ingreso a la universidad } & Inmediato & 33 \\
\hline & Posterior & 34 \\
\hline \multirow{2}{*}{ Educación de los padres } & Secundaria & 10 \\
\hline & Superior & 57 \\
\hline \multirow{2}{*}{ Procedencia escolar } & Estatal & 5 \\
\hline & Particular & 62 \\
\hline \multirow{2}{*}{ Categoría de pago } & A-B-C & 18 \\
\hline & D-E-F & 49 \\
\hline Total & & 67 \\
\hline
\end{tabular}


Los ítems del área de cálculo son nueve y contienen 27 preguntas; se orientan a explorar algunas de las destrezas y habilidades fundamentales para el manejo de los números en el continuo matemático.

En primer lugar, el ítem C-III explora las estimaciones o anticipaciones de resultado de operaciones, esta representación aporta un control metacognitivo muy importante.

En segundo lugar, las exploraciones vinculadas a las posiciones numéricas, que demuestran el dominio de la "escritura de numerales", esos ítems son: $\mathrm{C}-\mathrm{VI}$, transformaciones numéricas, $\mathrm{y}$ C-VIII, posiciones numéricas.

En tercer lugar, otros ítems se dirigen a explorar las relaciones de los núme- ros con estructuras gráficas, son los ítems C-XIII, factorización gráfica; CIX, ábaco; $\mathrm{C}-\mathrm{XV}$, números en recta; $\mathrm{y}$ C-XX, números a recta.

Finalmente, en cuarto lugar, los ítems C-XIV y C-XVII se dirigen a explorar relaciones de lo que piagetanamente denominaríamos "operaciones multiplicativas": factorización y fracciones.

Los ítems del área de problemas son cuatro, con sus correspondientes tres preguntas cada uno. Esos ítems son: P$\mathrm{X}$, comprensión y clasificación matemática de enunciados narrativos; P-XI, traducir enunciados narrativos a ecuaciones; P-XVIII, generar problemas narrativos a partir de ecuaciones; y PXIX, identificar las proposiciones relevantes de enunciados narrativos.

Tabla $\mathbf{N}^{\circ} 2$

Dimensiones exploradas por cada ítem

\begin{tabular}{llll}
\hline İtem & Dimensión & Ítem & Dimensión \\
\hline & & & \\
O-I & Organización del conocimiento & C-VIII & Posición numérica \\
O-II & Operaciones aritméticas & C-IX & Ábaco \\
O-IV & Lectura de gráficos & C-XIII & Factorización gráfica \\
O-V & Distribución normal & C-XIV & Fracciones \\
O-VII & Combinaciones & C-XV & Números en recta \\
O-XII & Probabilidades & C-XVII & Identificar primos \\
O-XVI & Proporciones & C-XX & Números a recta \\
O-XXI & Seriación & P-X & Enunciados narrativos \\
C-III & Estimaciones & P-XI & Traducir ecuaciones \\
C-VI & Transformaciones & P-XVIII & Generar problemas \\
& & P-XiX & Enunciados relevantes \\
\hline
\end{tabular}


Todos los ítems tratan de explorar la relación entre el lenguaje narrativo natural y los conocimientos y habilidades matemáticos de los sujetos.

\section{Procedimiento}

Se estructuró un cuadernillo con las 63 preguntas, con los ítems seriados secuencialmente del I al XXI y una hoja de respuestas ad hoc. Los sujetos fueron examinados en situación grupal convocados por sus autoridades académicas. No conocían la finalidad última de la investigación, salvo que se "quería conocer cuánto manejaban las matemáticas básicas los alumnos iniciales para generar apoyos y ayudas a todos los estudiantes".

Se les señaló que las pruebas eran anónimas, porque no se trataba de notas, pero que sí se les pedía esforzarse en resolver lo mejor que pudieran los sencillos problemas que se les presentarían y resolverlos no requería de mayores estudios que los que ya tenían. Se aplicaron las pruebas por separado a los sujetos de alto y bajo rendimiento, aunque éstos, por supuesto, ignoraban a qué grupo pertenecían. La calificación fue muy sencilla: Un punto la respuesta correcta, 0 punto la errada. En algunos ítems, como por ejemplo PXVIII, la estructura del propio ítem exigió implementar una calificación intermedia de medio punto.

\section{Resultados}

\section{Confiabilidad del instrumento}

Para analizar la confiabilidad del instrumento se le sometió al procesamiento por la escala Alpha. Los resultados se presentan en la tabla $\mathrm{N}^{\circ} 3$ organizados por áreas e ítems. Los coeficientes Alpha de la prueba por área son de $0=$ $0.55 ; \mathrm{C}=0.80$ y $\mathrm{P}=0.64$. Si se eliminan los ítems 0-I y 0-V del área 0 y $\mathrm{P}$ XIX del área $P$, ascienden los coeficientes Alpha parciales a $0=0.61$ y $\mathrm{P}=$ 0.70 que añadido a $\mathrm{C}=0.80$ las hace satisfactorias.

Los coeficientes Alpha de los puntajes totales son $0=0.68 ; \mathrm{C}=0.82$ y $\mathrm{P}=$ 0.69. El coeficiente Alpha, del puntaje total reducido es 0.79 . En resumen, de los 21 ítems explorados 18 tienen una buena confiabilidad y tres son de confiabilidad reducida.

\section{Validez del instrumento}

Para la exploración de la validez del instrumento se aplicaron tres pruebas: análisis factorial, análisis canónico discriminante y análisis de jerarquía, para ver la contribución de cada área al puntaje de los sujetos. 
Tabla $\mathbf{N}^{\circ} 3$

Escala Alpha de confiabilidad del instrumento

\begin{tabular}{|c|c|c|}
\hline Ítem & Corr. & Alpha \\
\hline $\mathrm{OY}$ & .1459 & .5586 \\
\hline O II & .2801 & $.5201^{*}$ \\
\hline O IV & .4339 & $.4469^{*}$ \\
\hline O V & .0395 & .6027 \\
\hline O VII & .2514 & $.5258^{*}$ \\
\hline O XII & .2574 & $.5224^{*}$ \\
\hline $\mathrm{OXVI}$ & .2585 & $.5207^{*}$ \\
\hline O XXI & .5064 & $.4103^{*}$ \\
\hline O total & (8 ítems) & $.5523^{*}$ \\
\hline O reducido & (6 ítems) & $.6079^{*}$ \\
\hline Ítem & Corr. & Alpha \\
\hline C III & .3475 & $.7930^{*}$ \\
\hline $\mathrm{C} \mathrm{VI}$ & .3803 & $.7893^{*}$ \\
\hline C VIII & .4963 & $.7763^{*}$ \\
\hline C IX & .3868 & $.7883^{*}$ \\
\hline C XIII & .5776 & $.7669^{\star}$ \\
\hline C XIV & .5704 & $.7641^{*}$ \\
\hline$C \times V$ & .6020 & $.7612^{*}$ \\
\hline C XVII & .4510 & $.7814^{*}$ \\
\hline$c x X$ & .5848 & $.7621^{\star}$ \\
\hline C Total & (9 ítems) & $.7963^{*}$ \\
\hline Ítem & Corr. & Alpha \\
\hline$P X$ & .3909 & $.5986^{*}$ \\
\hline P XI & .4850 & $.5300^{\star}$ \\
\hline PXVIII & .6551 & $.3805^{\star}$ \\
\hline$P X I X$ & .1895 & .7033 \\
\hline C Total & (4 ítems) & $.6446^{\star}$ \\
\hline C Reducido & (3 ítems) & $.7033^{*}$ \\
\hline OPT & .7206 & $.6846^{*}$ \\
\hline СРТ & .7227 & $.8185^{\star}$ \\
\hline PPT & .7417 & $.6906^{*}$ \\
\hline Total reducido & & $.7865^{\star}$ \\
\hline
\end{tabular}

* Ítem significativo

En la tabla $\mathrm{N}^{\circ} 4$, el análisis factorial nos presenta una excelente prueba de validez. La comunalidad de las áreas con respecto a un único factor es, respectivamente, para $0=.78, \mathrm{C}=.77 \mathrm{y} \mathrm{P}$ $=.80$, con una varianza explicada del $78,4 \%$. Este resultado expresa una alta validez de constructo del instrumento.

Esta validez se ve confirmada por los resultados del análisis canónico discriminante (tabla $\mathrm{N}^{\circ}$ 5) donde el agrupamiento de los sujetos en función del rendimiento con los puntajes de la prueba es correcto en un 92,54\%; correspondiendo en esa predicción $85,2 \%$ a los de rendimiento alto y $97,58 \%$ a los de rendimiento bajo.

Finalmente, en la tabla $\mathrm{N}^{\circ} 6$ aparece el análisis de jerarquía que nos ofrece el peso con el que cada área contribuye al puntaje total. $\mathrm{C}=1,8 ; 0=1,7$ y $\mathrm{P}=$ 1,3. Es decir, tenemos una secuencia contribucional en el orden siguiente: cálculo, operaciones y problemas.

Tabla $N^{\circ} 4$

Análisis factorial (validez)

\begin{tabular}{lll}
\hline Comunalidad & Factor & $\begin{array}{l}\text { Varianza } \\
\text { explicada }\end{array}$ \\
\hline 0.78318 & 1 & 78.4 \\
C .76865 & & \\
P.79900 & & \\
\hline
\end{tabular}

Tabla No 5

Análisis canónico discriminante (validez)

\begin{tabular}{lcc}
\hline Grupo & \multicolumn{2}{c}{ Predicción (\%) } \\
\hline & $(1)$ & $(2)$ \\
(1) Rendimiento alto & 85,2 & 14,8 \\
(2) Rendimiento bajo & 2,58 & 97,58 \\
\hline
\end{tabular}

Agrupamiento correcto $92,54 \%$ 
Tabla $N^{\circ} 6$

Análisis de jerarquía (contribución)

Media
$C=1.809$
$0=1.736$
$P=1.336$

\section{Los rasgos grupales}

Se han explorado, además del rendimiento, los siguientes rasgos grupales: edad, sexo, carrera, lugar de nacimiento, repitencia, ingreso, educación de los padres, procedencia escolar y categoría de pago. En la tabla $\mathrm{N}^{\circ} 7$ se presentan los resultados del análisis de regresión múltiple respecto del papel predictivo de estos factores referentes a los puntajes de la prueba. El único factor predictor es la diferenciación de rendimiento académico en alto y bajo con un coeficiente Beta de .74 y una significación de 6.32; ningún otro factor es significa- tivamente predictor de puntuaciones. Este resultado se confirma con los de la tabla $\mathrm{N}^{\circ} 8$, que resume los análisis factoriales de varianza de los puntajes obtenidos tomando los rasgos sociales como fuente de varianza y, además, como variable asociada al nivel de rendimiento.

Cuando el rasgo grupal es tomado como fuente de varianza ningún coeficiente es significativo; en cambio cuando se hace análisis de varianza a cada grupo, constituido por cada rasgo social, y se le analiza teniendo como variable asociada al nivel de rendimiento alto y bajo, esta variable es significativamente alta en todos los casos. Estos resultados nos confirman que con respecto a las puntuaciones obtenidas, los rasgos sociales no tienen carácter predictor.

Tabla $\mathbf{N}^{\circ} 7$

Papel predictivo de los rasgos poblacionales por análisis de regresión múltiple multivariado con interacción de todas las variedades

\begin{tabular}{|c|c|c|c|c|c|c|c|c|}
\hline \multirow[t]{2}{*}{ Variable } & \multicolumn{2}{|c|}{$\begin{array}{l}\text { Puntaje } \\
\text { operatorio }\end{array}$} & \multicolumn{2}{|c|}{$\begin{array}{c}\text { Puntaje } \\
\text { cálculo }\end{array}$} & \multicolumn{2}{|c|}{$\begin{array}{l}\text { Puntaje } \\
\text { problemas }\end{array}$} & \multicolumn{2}{|c|}{$\begin{array}{c}\text { Puntaje } \\
\text { total }\end{array}$} \\
\hline & Beta & $\mathrm{T}$ & Beta & $\mathrm{T}$ & Beta & $\mathrm{T}$ & Beta & $\mathrm{T}$ \\
\hline Rendimiento & .58 & $3.95^{*}$ & .75 & $6.70^{*}$ & .56 & $3.70^{*}$ & .74 & $6.32^{*}$ \\
\hline Edad & -.17 & 1.14 & .02 & -.15 & .14 & .92 & .10 & .80 \\
\hline Sexo & -.08 & .75 & .03 & .43 & .01 & .11 & .01 & .07 \\
\hline Carrera & .11 & .84 & .03 & .38 & .12 & .89 & .08 & .81 \\
\hline Lugar Nac. & .06 & .54 & -.07 & -.93 & .07 & .64 & .01 & .09 \\
\hline Repitencia & .10 & .92 & -.15 & -1.87 & .13 & 1.22 & .15 & 1.75 \\
\hline Ingreso & .14 & 1.11 & -.11 & -1.15 & .07 & .55 & .00 & .04 \\
\hline Educ. Padres & .03 & .26 & -.19 & -2.22 & .07 & .60 & .12 & 1.31 \\
\hline Procedencia & -1.14 & -1.39 & .02 & .26 & .08 & .73 & .05 & .60 \\
\hline Categoría & -.06 & -.56 & -.04 & .55 & .03 & .30 & .05 & .60 \\
\hline
\end{tabular}

${ }^{*}$ Significativo $<.000$ 
Tabla $N^{\circ} 8$

Análisis factoriales de varianza de los puntajes por rasgos sociales como fuente y como variable asociada al nivel de rendimiento

\begin{tabular}{lrr}
\hline $\begin{array}{l}\text { Rasgo } \\
\text { grupal }\end{array}$ & Fuente & $\begin{array}{c}\text { Nivel de } \\
\text { rendimiento }\end{array}$ \\
\hline Edad & 1.015 & $98.210^{*}$ \\
Sexo & .014 & $95.675^{*}$ \\
Carrera & .229 & $95.953^{*}$ \\
Lugar de nac. & .044 & $88.686^{*}$ \\
Repitencia & 2.762 & $101.068^{*}$ \\
Ingreso & .009 & $94.233^{*}$ \\
Educ. padres & .922 & $97.829^{*}$ \\
Procedencia & .549 & $95.577^{*}$ \\
Categoría & .004 & $98.445^{*}$ \\
\hline
\end{tabular}

${ }^{*}$ significativo $<.000$

Sin embargo, los resultados de las pruebas $t$ de carácter inferencial sólo coinciden parcialmente en los resultados anteriores. Obsérvese que estos resultados exploran las diferencias de rendimientos de grupos. Los grupos que difieren significativamente en sus puntuaciones son los de rendimiento y también los de edad y carrera.

En la tabla $\mathrm{N}^{\circ} 9$ se presentan los puntajes por área $\mathrm{O}, \mathrm{C}$ y $\mathrm{P}$ y totales por grupos de rendimiento y los otros nueve factores sociales. La edad diferencia los rendimientos hacia abajo de los jóvenes entre 18-20 años, los estudiantes menores de 18 y mayores de 20 tienen mejores rendimientos. La carrera a estudiar, letras o ciencias también tienen

Tabla $\mathbf{N}^{\circ} 9$

Análisis inferencial de las puntuaciones promedio por rasgos grupales (prueba t)

\begin{tabular}{|c|c|c|c|c|c|c|c|c|c|}
\hline & & $\mathrm{Ot}$ & C & $\mathrm{t}$ & $P$ & $\mathrm{t}$ & T & $\mathrm{t}$ & \\
\hline \multirow{2}{*}{ Rendimiento } & Alto & 12.44 & $6.10^{*}$ & 21.48 & $10.09^{*}$ & 5.85 & $5.89^{*}$ & 39.78 & $9.85^{*}$ \\
\hline & Bajo & 9.05 & & 12.78 & & 2.76 & & 24.59 & $15.85^{*}$ \\
\hline \multirow{3}{*}{ Edad (1) } & Menos 18 & 12.00 & $8.29^{*}$ & 20.26 & $15.39^{*}$ & 5.42 & 8.75 & 37.68 & \\
\hline & $18-20$ & 9.45 & & 13.88 & 3.10 & & 26.43 & & \\
\hline & Más 20 & 12.14 & & 20.46 & & 5.86 & & 38.46 & \\
\hline \multirow[t]{2}{*}{ Sexo } & Masc. & 10.30 & -.49 & 16.29 & .01 & 4.00 & -.03 & 30.59 & -.14 \\
\hline & Fem. & 10.66 & & 16.27 & & 4.02 & & 30.96 & \\
\hline \multirow[t]{2}{*}{ Carrera } & Ciencias & 11.10 & $3.47^{*}$ & 17.60 & $3.34^{*}$ & 4.63 & $3.39^{*}$ & 33.33 & $3.87^{*}$ \\
\hline & Letras & 8.68 & & 12.96 & & 2.45 & & 24.09 & \\
\hline \multirow{2}{*}{ Lug.Nac. } & Lima & 10.41 & .49 & 15.83 & -1.51 & 4.01 & -.31 & 30.25 & -.91 \\
\hline & Prov. & 10.89 & & 18.78 & & 3.72 & & 33.39 & \\
\hline \multirow[t]{2}{*}{ Repitencia } & Sí & 9.25 & -1.27 & 13.06 & -1.79 & 2.75 & -1.48 & 25.06 & -1.78 \\
\hline & No & 10.58 & & 16.72 & & 4.18 & & 31.47 & \\
\hline \multirow[t]{2}{*}{ Ingreso } & Inmediato & 11.27 & 2.58 & 17.31 & 1.52 & 4.68 & 2.16 & 33.27 & 2.19 \\
\hline & Posterior & 9.59 & & 15.29 & & 3.35 & & 28.23 & \\
\hline \multirow[t]{2}{*}{ Educ.Padres } & Secund. & 10.10 & -.39 & 12.68 & -2.32 & 3.15 & -1.14 & 25.93 & -1.72 \\
\hline & Superior & 10.47 & & 16.92 & & 4.16 & & 31.55 & \\
\hline \multirow[t]{2}{*}{ Procedencia } & Estatal & 8.20 & -1.89 & 14.95 & -.56 & 2.50 & -1.36 & 25.65 & .228 \\
\hline & Particular & 10.60 & & 16.39 & & 4.13 & & 31.12 & \\
\hline \multirow{2}{*}{ Categoría } & $A-B-C$ & 9.94 & -.84 & 15.79 & -.44 & 3.83 & -.33 & 29.57 & -.58 \\
\hline & D-E-F & 10.59 & & 16.46 & & 4.07 & & 31.13 & \\
\hline
\end{tabular}

*Significación <.00

(1) Valor F 
un papel. Los alumnos que se orientan a ciencias tienen mejores rendimientos que los que se orientan a letras.

\section{Las estrategias exploradas}

En la tabla $\mathrm{N}^{\circ} 10$ presentamos los resultados de las pruebas de inferencia $t$ aplicadas a cada uno de los ítem explorados, ordenados por cada área de estrategia $\mathrm{O}, \mathrm{C}$ y $\mathrm{P}$. En la tabla $\mathrm{N}^{\circ} 9$ ya hemos visto las pruebas $t$ correspondientes a las puntuaciones parciales $\mathrm{O}$, $\mathrm{C}$, P y al puntaje total; en esta tabla se presentan las pruebas $t$ de los ítems.
Hay cinco ítems que presentan diferencias significativas, tres de ellos en $\mathrm{O}$ : distribuciones, combinaciones $\mathrm{y}$ probabilidades. Un ítem en C: estimaciones, y un ítem en P: enunciados relevantes.

Todos los otros ítems presentan diferencias significativas. Estos resultados nos dan un perfil del estudiante de buen rendimiento y del estudiante de bajo rendimiento académico pero, además, nos identifican las dificultades de ambos tipos de estudiantes, como lo analizaremos en los próximos parágrafos.

Tabla $\mathbf{N}^{\circ} 10$

Prueba inferencial $t$ correspondiente a cada área de ítem en los grupos de alto y bajo rendimiento

\begin{tabular}{llccc}
\hline İtem & \multicolumn{1}{c}{ Dimensión } & Prom. g. alto & Prom g. bajo & $\mathrm{t}$ \\
\hline & & & & \\
O-I & Org. del conocimiento & 1.81 & 1.40 & $2.03^{*}$ \\
O-II & Operaciones aritméticas & 3.00 & 2.52 & $3.65^{*}$ \\
O-IV & Lectura de gráficos & 2.29 & 1.70 & $2.58^{*}$ \\
O-V & Distribución normal & 1.48 & 1.55 & -.29 \\
O-VII & Combinaciones & .89 & .78 & .76 \\
& Probabilidades & .96 & .78 & 1.11 \\
O-XVI & Proporciones & 2.89 & 1.93 & $5.75^{*}$ \\
O-XXI & Seriación & 2.41 & 1.35 & $4.76^{*}$ \\
C-III & Estimaciones & 1.85 & 1.45 & 1.85 \\
C-VI & Transformación de números & 2.67 & 2.15 & $3.10^{*}$ \\
C-VIII & Posición numérica & 2.70 & 1.75 & $3.52^{*}$ \\
C-IX & Ábaco & 2.78 & 2.05 & $4.01^{* *}$ \\
C-XIII & Factorización gráfica & 2.11 & 1.03 & $6.69^{* *}$ \\
C-XIV & Fracciones & 2.81 & 1.20 & $8.27^{* *}$ \\
C-XV & Números en recta & 2.28 & 1.28 & $4.84^{* *}$ \\
C-VII & Identificar primos & 1.81 & .88 & $3.98^{* *}$ \\
C-XX & Números a recta & 2.93 & .99 & $5.71^{* *}$ \\
P-X & Enunciados narrativos & 1.56 & .95 & $2.51^{*}$ \\
P-XI & Traducción de ecuaciones & 2.33 & 1.05 & $5.56^{* *}$ \\
P-XVIII & Generar problemas & 1.86 & .76 & $4.94^{* *}$ \\
P-XIX & Enunciados relevantes & .76 & .76 & -.02 \\
\hline
\end{tabular}

* Significativo $<.05$

** Significativo $<.000$ 


\section{Discusión}

\section{Los factores grupales}

Los resultados nos indican claramente que la pertenencia de los sujetos a diversos subgrupos poblacionales no tiene valor predictivo sobre su puntuación en la prueba; es decir, la actuación matemática no es dependiente ni del sexo, procedencia escolar o alguno de los factores explorados. Sin embargo, la edad y la carrera presentan promedios consistentemente diferentes a las pruebas $t$. Por un lado, la debilidad en la actuación se relaciona con la edad, los jóvenes de 18 a 20 años tienen en promedio puntuaciones más bajas que los jóvenes menores de 18 y mayores de 20 . Es posible que intervengan aquí factores vinculados con la estabilidad emocional y con la dinámica motivacional respecto de las "performances" de los jóvenes más maduros. Los jóvenes intermedios (18-20) están saliendo de la adolescencia y estarían con una mayor labilidad oréctica.

\section{Los perfiles de rendimiento}

Los resultados obtenidos nos permiten hacer un perfil del dominio de estrategias que corresponden tanto al alumno de alto rendimiento como al de bajo rendimiento y un tercer perfil centrado en las dificultades que ambos grupos afrontan.

El perfil del sujeto con alto rendimiento sería el siguiente: sus estrate- gias operatorias de clasificación de clases; operaciones aritméticas básicas; operaciones de medición y sus operaciones de seriación dependientes de estrategias lógico-concretas están consolidadas y las aplica con relativa fluidez, especialmente en el reconocimiento de las operaciones aritméticas básicas. En el aspecto de los esquemas operatorios lógico-formales domina el esquema de proporciones y afronta esas tareas adecuadamente. En lo que respecta al cálculo hay un consistente dominio de las posiciones numéricas, de las relaciones entre esquemas gráficos y valores numéricos y algo más débil de las operaciones factoriales y fraccionarias. En el área de problemas, nuestro sujeto vincula con relativa consistencia el discurso del lenguaje natural con el discurso matemático y se aproxima con relativa comprensión a los enunciados narrativos de aspectos matemáticos.

El perfil del estudiante con bajo rendimiento es el siguiente: una fuerte deficiencia en los dominios operatorios lógico-concretos aplicados a las matemáticas: clasificaciones muy débiles, bajo reconocimiento de operaciones numéricas elementales, operaciones de medición y seriación muy débiles. Igual debilidad se presenta en el área de las proporciones, esquema operatorio relativamente aún débil. En lo que respecta al cálculo, tiene relativa debilidad en el dominio de las posiciones numéricas, en las relaciones entre dimensiones gráficas y valores numéri- 
cos y además la factorización y operaciones con fracciones muy debilitadas. En lo que respecta a los problemas, afronta serias dificultades en relacionar los enunciados del lenguaje natural con el discurso matemático, realizando a muy bajo nivel las traducciones pertinentes. Finalmente, hay debilidad en su aproximación comprensiva a los enunciados narrativos desde el punto de vista matemático.

El perfil de dificultades común a ambos grupos de alto y bajo rendimiento es el siguiente: debilidad en los esquemas operatorios lógico-formales de probabilidad, distribución normal y combinaciones en el área que hemos denominado operatoria. En el área de cálculo, el problema común son las estimaciones, con relativa debilidad compartida; este aspecto del pensamiento matemático está vinculado a los procesos metacognitivos. En el aspecto de problemas, hay en ambos grupos mucha debilidad en identificar lo relevante de un enunciado narrativo desde la perspectiva del planteo de un problema matemático; es un proceso vinculado a la comprensión más profunda de un texto: destacar la idea principal.

Finalmente, queremos hacer referencia a las observaciones informales que hemos registrado sobre la alta tasa de conductas de rechazo y aversión que se presentan, especialmente en los sujetos de rendimiento bajo cuando tienen que afrontar tareas matemáticas.

\section{REFERENCIAS}

Barody, A. (1994). El pensamiento matemático de los niños. Madrid: Visor.

Brissiaud, E. (1993). El aprendizaje del cálculo. Madrid: Visor.

Deaño, D. (1993). Conocimiento lógicomatemático en la escuela infantil. Madrid: Cepe.

Dienes, Z. (1970). La construcción de las matemáticas. Barcelona: Vicens-Vives.

Dienes, Z. (1971). El aprendizaje de la matemática. Buenos Aires: Estrada.

Dienes, Z. (1971). La potencia de la matemática. Buenos Aires: Estrada.

Dienes, Z. (1975). Enseñanza y aprendizaje de la matemática. Buenos Aires: Paidós.

Gagné, E. (1991). La psicología cognitiva del aprendizaje escolar. Madrid: Visor.

Gagné, R. (1987). Las condiciones del aprendizaje. México, DF: Prentice Hall.

González M. (1998). “Comprensión lectora en estudiantes universitarios iniciales". Persona (Universidad de Lima), 1, 43-65.

González M. (1998). "Psicología educacional de las matemáticas". Revista de Investigaciones Psicológicas. (Universidad Nacional Mayor de San Marcos), 2, 9-40.

Holloway, G. (1969). Piaget. Buenos Aires: Paidós.

Holloway, G. (1969). Concepción del espacio en el niño según Piaget. Buenos Aires: Paidós.

Hughes, M. (1987). Los niños y los números. Barcelona: Planeta. 
Inhelder, B. \& J. Piaget, (1972). De la lógica del niño a la lógica del adolescente. Buenos Aires: Paidós.

Isaacs, N. (1967). Nueva luz sobre la idea de número en el niño. Buenos Aires: Paidós.

Jurado, C. (1993). Didáctica de la matemática. Quito: Abya-Vala.

Kamii, C. (1993). El niño reinventa la aritmética. Madrid: Visor.

Lave, J. (1991). La cognición en la práctica. Buenos Aires: Paidós.

Laurence E. \& otros, (1968). La comprensión del número y la educación del niño según Piaget. Buenos Aires: Paidós.

Lovell, K. (1969). Didáctica de las matemáticas. Madrid: Morata.

Maza, C. (1995). Aritmética y representación. Barcelona: Paidós.

Orton, A. (1990). Didáctica de las matemáticas. Madrid: Morata.

Piaget, J. \& A. Szeminska, (1967). Génesis del número en el niño. México: Guadalupe.

Piaget, J. \& otros, (1965). La enseñanza de las matemáticas. Madrid: Aguilar.

Piaget, J. \& otros, (1983). La enseñanza de las matemáticas modernas. Madrid: Alianza.

Resnick L. \& W. Ford, (1990). La enseñanza de las matemáticas y sus fundamentos psicológicos. Barcelona: Paidós.

Resrick L. \& L. Klopter, (1996). Currículum y cognición. Buenos Aires: Aique.

Skemp, R. (1999). Psicología de la enseñanza de la matemática. Madrid: Morata. 